EGU2020-13099

https://doi.org/10.5194/egusphere-egu2020-13099

EGU General Assembly 2020

(c) Author(s) 2020. This work is distributed under

the Creative Commons Attribution 4.0 License.

\title{
Deconvolution of Laboratory IR Spectral Reflectance Measurements of Olivine-Pyroxene Mineral Mixtures.
}

\author{
Karin E. Bauch ${ }^{1}$, Iris Weber ${ }^{1}$, Maximilian P. Reitze ${ }^{1}$, Andreas Morlok ${ }^{1}$, Harald Hiesinger ${ }^{1}$, \\ Aleksandra N. Stojic ${ }^{1}$, and Jörn Helbert ${ }^{2}$ \\ ${ }^{1}$ Westfälische Wilhelms-Universität Münster, Institut für Planetologie, Münster, Germany (karin.bauch@uni-muenster.de) \\ ${ }^{2}$ DLR-Institute for Planetary Research, Rutherfordstrasse 2, 12489 Berlin, Germany
}

The imaging spectrometer MERTIS (Mercury Radiometer and Thermal Infrared Spectrometer) is part of the payload of ESA/JAXA's BepiColombo mission, launched in $2018[1,2]$. The instrument consists of an IR-spectrometer and radiometer, which will observe the surface in the wavelength range of $7-14 \mu \mathrm{m}$ and $7-40 \mu \mathrm{m}$, respectively. In preparation of the mission, we are investigating Mercury analog minerals at the IRIS (Infrared and Raman for Interplanetary Spectroscopy) laboratory of the Institut für Planetologie at the Westfälische Wilhelms-Universität Münster. We study typical rock-forming minerals, e.g., pyroxenes, olivines, and feldspars, as well as mineral mixtures.

Here we present results of a deconvolution model used to quantify mineral specific abundances of mineral mixtures [4,5]. Planetary surfaces are composed of a variety of different minerals, therefore the obtained spectral data reflects a mixture of these minerals. In order to quantify the mineral abundances a non-linear unmixing model is necessary. Our model is based on the Hapke reflectance theory [6-8] and is applied to data obtained at the IRIS laboratory [9]. Results of olivine and pyroxene mixtures, as well as grain size mixtures, will be presented at the meeting.

We used olivine $\left(\mathrm{Fo}_{91}\right)$ from Dreiser Weiher, Germany, and pyroxene $\left(\mathrm{En}_{87}\right)$ from Bamble, Norway and a range of mineral mixtures for IR measurements. Samples are sieved in grain size fractions of $<25 \mu \mathrm{m}, 25-63 \mu \mathrm{m}, 63-125 \mu \mathrm{m}$, and $125-250 \mu \mathrm{m}$. For the mineral mixing analysis presented here, we focus on the $63-125 \mu \mathrm{m}$ fraction, which was also used by $[10,11]$ for further investigations. Samples are analyzed by a Bruker Vertex 70v spectrometer with an A513 variable mirror reflectance stage for various incidence/emergence angles. A total of 512 single channel scans of the sample and the background (diffuse gold standard INFRAGOLD ${ }^{\mathrm{TM}}$ ) were accumulated to ensure a high signal-tonoise ratio.

The pure pyroxene and olivine spectra clearly show characteristic Christiansen features and Reststrahlen bands for all applied geometries and increasing phase angles result in decreased intensities. The reflectance increases from pyroxene and pyroxene-rich mixtures to olivine and olivine-rich mixtures. Moreover, the olivine-rich mixtures exhibit more olivine reflectance features, compared to pyroxene-rich mixtures [11]. 
Our studies of pyroxene grain size analysis focus on pyroxene mixtures of $50 \%$ fine/50\%coarse and $30 \%$ fine/70\%coarse material. Generally, the intensities increase with increasing grain sizes. The transparency feature is evident for small grain sizes and the $50 \%$ fine $/ 50 \%$ coarse mixture.

At IRIS laboratory, we will further investigate planetary analog material and their mineral mixtures applying various analytical techniques. With these data we are establishing a database that will enable the correct interpretation of MERTIS results.

This work has been funded by DLR grant 50 QW 1701 in the framework of the BepiColombo mission.

[1] Hiesinger H. et al. (2010) PSS58, 144-165. [2] Benkhoff J. et al. (2010) PSS58, 2-20. [3] Grumpe A. et al (2017) Icarus299, 1-14. [4] Rommel D. et al. (2017) Icarus284, 126-149. [5] Hapke B. (1981), JGR86(B4), 3039-3054. [6] Hapke B. (2002), Icarus 157, 523-534. [7] Hapke B. (2012), $2^{\text {nd }}$ Cambr. Univ. Press., NY. [8] Bauch, K.E. et al. (2019) LPSC L, Abstract\#2521. [9] Weber I. et al. (2019) LPSC L, Abstract\#2326. [10] Weber, l. et al. (2020) LPSC LI, Abstract\#1889. 